\title{
An Outbreak of Cholera Associated with an Unprotected Well in Parbatia, Orissa, Eastern India
}

\author{
Amitav Das', P. Manickam', Yvan Hutin², B.B. Pal'², G.P. Chhotray², S.K. Kar², and M.D. Gupte' \\ 'National Institute of Epidemiology (ICMR), Chennai, Tamil Nadu, India and \\ ${ }^{2}$ Regional Medical Research Centre (ICMR), Bhubaneswar, Orissa, India
}

\begin{abstract}
In November 2003, an outbreak (41 cases; attack rate-4.3\%; no deaths) of severe diarrhoea was reported from a village in Orissa, eastern India. Thirteen of these cases were hospitalized. A matched case-control study was conducted to identify the possible exposure variables. Since all wells were heavily chlorinated immediately after the outbreak, water samples were not tested. The cases were managed symptomatically. Descriptive epidemiology suggested clustering of cases around one public well. Vibrio cholerae El Tor O1, serotype Ogawa was isolated from four of six rectal swabs. The water from the public well was associated with the outbreak (matched odds ratio: 12; 95\% confidence interval 1.2-44.1). On the basis of these conclusions, access to the well was barred immediately, and it was protected. This investigation highlighted the broader use of field epidemiology methods to implement public-health actions guided by epidemiologic data to control a cholera epidemic.
\end{abstract}

Key words: Case-control studies; Cholera; Disease outbreaks; Sanitation; Vibrio cholerae; Water supply; India

\section{INTRODUCTION}

Cholera is an infectious, acute watery diarrhoea caused by Vibrio cholerae serogroup O1 and O139 $(1,2)$. V. cholerae produces a toxin that stimulates the secretion of water and electrolytes in the intestinal tract. Patients with cholera may suffer from acute watery diarrhoea, vomiting, and dehydration but rarely present with fever (3). The El Tor biotype of $V$. cholerae is less severe than classical cholera (3). Worldwide, cholera is transmitted through a number of sources, including water and food (1).

India remains endemic for cholera, particularly in its eastern part (4). V. cholerae O139 emerged in the country since 1992 (5) but does not account for the majority of cases (6). Clusters of acute diarrhoea are common but investigations are difficult to conduct. First, many clusters of diarrhoea are not investigated using laboratory methods that could lead to the identification of an aetiological agent. Thus, cholera, including the El Tor biotype, may be

Correspondence and reprint requests should be addressed to:

Dr. P. Manickam

Scientist B

National Institute of Epidemiology (ICMR)

Chennai, Tamil Nadu 600077

India

Email: manickamp@gmail.com

Fax: +91-44-26820464 under-recognized, and many outbreaks are simply recorded as 'diarrhoea outbreaks' (7). Second, epidemiological investigations are solely descriptive and do not include the typical sequence of formulating and testing of hypothesis that is classical of field epidemiology. As a result, sources of infections are difficult to identify, and preventive measures cannot be implemented. In 2001, the National Institute of Epidemiology (NIE) of the Indian Council of Medical Research (ICMR) initiated the Field Epidemiology Training Programme (FETP) that placed epidemiologists in training in various states of India. This initiative led to field investigations of various types of outbreaks that generated practical information for action.

On 14 November 2003, a primary health centre in Dhenkanal district, Orissa, Eastern India, reported a cluster of acute, severe diarrhoea with dehydration among adults in the village of Parbatia to the district public-health authorities. The population of the village in 2003 was 946 . Cholera was suspected in the diagnosis. On 15 November 2003, an epidemiologist from the FETP assigned to the state of Orissa initiated an investigation and arrived in the village in the morning to investigate the outbreak. The objectives of the investigation were to characterize the agent, describe the persons affected, identify the source of infection, and formulate practical recommendations for control. 


\section{MATERIALS AND METHODS}

\section{Descriptive epidemiology}

A case of diarrhoea was defined as the occurrence of more than three watery stools a day among residents of the village in November 2003. This case definition was consistent with the recommendations of the Orissa Multi Disease Surveillance System (OMDSS). The principal investigator, local health workers, and laboratory technicians searched actively for cases door-to-door in the village. They collected personal history, including symptoms, from case-patients and created a line listing. They constructed an epidemic curve to describe the development of the outbreak over time, plotted cases on a map to understand the geographical distribution of the disease, and calculated the incidence by age and sex using population denominators collected during the door-to-door case search. Hypotheses were generated based on characteristics of person, time and place of the outbreak. They could identify an initial patient with a date of onset that preceded all others and interviewed the whole family to determine whether this patient could have constituted a source of infection for the community.

\section{Matched case-control study}

To test the main hypothesis regarding the possible source of infection, our team conducted a matched case-control study. All the case-patients identified (minus the initial, index patient, who we suspected to be the source) were included during the doorto-door case search using the same case definition. For each case-patient, two neighbourhood control subjects matched for sex, age (one year), and socioeconomic status were recruited. Only those control-subjects who apparently did not have cholera were included. A standardized, close-ended questionnaire was used for collecting information on potential exposures from the subjects. We defined our referent exposure period as three days preceding the illness. Matched odds ratios (MORs) were calculated for discordant pairs and their 95\% confidence intervals (CIs) using the Epi Info software (version 6.04d) (CDC, Atlanta, GA, USA). The fraction of cases attributable to the exposure in the population was calculated using the classical formula: the proportion of cases exposed multiplied by the attributable fraction among the exposed [(odds ratio-1) divided by odds ratio)] (8).

\section{Laboratory procedure}

Rectal swabs were collected from a sample of casepatients selected at random before any treatment with antibiotics and were sent to the Regional Medical Research Centre (RMRC) of the ICMR in Bhubaneswar, the state capital. The laboratory conducted streak culture in alkaline nutrient agar for Vibrio and identified the agent using biochemical methods, serum agglutination, sheep red blood-cell haemolysis, and serotyping methods. We did not test for any antimicrobial resistance.

\section{Environmental investigations}

As the descriptive epidemiology and the hypothesis-generating interviews pointed to a contaminated well as the potential source of the outbreak, the water supply and sanitation situation was reviewed interviewing the index case-patient and community leaders and through observations.

\section{RESULTS}

\section{Descriptive epidemiology}

The surveillance data for the district and the village collected during 2001-2003 confirmed the unusual increase in incidence. The incidence in the primary health centre area in November 2003 was higher than that in the rest of the district and higher than that in the same area during the previous years. Further investigations ruled out recent population influx or any change in the reporting system. This episode was, thus, considered an outbreak.

Forty-one cases were identified from 40 casepatients among the 946 residents of the village (attack rate-4.3\%). There were no deaths. Of the 40 case-patients, $29 \%$ had vomiting, $47 \%$ had abdominal pain, and $32 \%$ had dehydration. None had blood in stools or fever.

The attack rate was high among adults, which was compatible with the diagnosis of cholera (Table).

Females had a slightly higher attack rate. There was an initial case on 10 November, followed by a rapid increase in the number of cases leading to a peak on 15 November and a progressive subsequent decrease, and the last case was on 21 November (Fig. 1). This led to suspect a common source of the outbreak, although the second part of the epidemic curve could have been stretched because of secondary person-to-person transmission. Of the 40 casepatients, $24(60 \%)$ were clustered around an unprotected well located close to the residence of the index case-patient (Fig. 2). Hypothesis-generating interviews with this index case-patient indicated that this person fell sick and soiled his clothes with diarrhoea. Thus, he left his house (shown with a 


\begin{tabular}{|lccc|}
\hline \multicolumn{4}{|c|}{$\begin{array}{l}\text { Table. Attack rates of acute diarrhoea by age and } \\
\text { sex, Parbatia, Orissa, India, 2003 }\end{array}$} \\
\hline $\begin{array}{l}\text { Characteristics } \\
\text { of patients }\end{array}$ & Population No. of cases & $\begin{array}{c}\text { Incidence } \\
\text { (\%) }\end{array}$ \\
\hline $\begin{array}{l}\text { Age (years) } \\
0-4\end{array}$ & 113 & 6 & 5 \\
$5-14$ & 190 & 4 & 2 \\
$15-24$ & 128 & 5 & 4 \\
$25-34$ & 144 & 5 & 3 \\
$35-44$ & 129 & 6 & 5 \\
$45-54$ & 88 & 4 & 4 \\
$55-64$ & 67 & 8 & 12 \\
$\geq 65$ & 87 & 3 & 3 \\
Sex & & & \\
Male & 481 & 17 & 3 \\
Female & 465 & 24 & 5 \\
Total & 946 & 41 & 4 \\
\hline
\end{tabular}

triangle on the map) and crossed the road to wash his soiled clothes in the unprotected well (Fig. 2). Thus, we generated the hypothesis that the source of the outbreak could have been drinking water from the contaminated well.

\section{Analytical epidemiology}

We recruited 40 matched case-control sets for the case-control study (excluding the initial casepatient). Their median age was 35 years, and 23 (58\%) of the 40 case-patients were female. Twenty-eight (70\%) of the 40 cases had drunk water from the suspected well compared to 23 (29\%) of the 80 matched controls (MOR: 12, 95\% CI 1.2-44.1). Further, if we exclude children aged less than five years and calculate the association, the MOR was 11.3 (95\% CI 1.2-42.1). Calculation of the popula- tion attributable fraction for both the MORs was identical, i.e. drinking water from the well could explain $64 \%$ of the cases.

\section{Laboratory investigations}

Four of the six rectal swabs grew $V$. cholerae El Tor O1, serotype Ogawa.

\section{Environmental investigations}

The well where the index case-patient washed his clothes were unprotected. It had no brim and no platform (Fig.3).

\section{DISCUSSION}

An outbreak of cholera struck the village of Parbatia in Orissa in November 2003. Unlike many similar outbreaks in the state, this outbreak was investigated using applied field epidemiology methods. These investigations suggested that the outbreak might have been caused by an unprotected well that had been contaminated by an initial casepatient who washed his clothes soiled with faeces while he was convalescent. In fact, $64 \%$ of the cases could be reasonably attributed to that contaminated well, and for the remaining case-patients, we did not have any specific explanation. However, a certain amount of person-to-person transmission may have occurred towards the second part of the outbreak.

Sources of contaminated water are often reported as sources of infection during outbreak of cholera in India $(9,10)$. The eighth Millennium Development Goal (MDG) of the United Nations proposed to halve the proportion of people who are unable to reach or to afford safe drinking-water between 1990 and 2015 (11). As per the United Nations

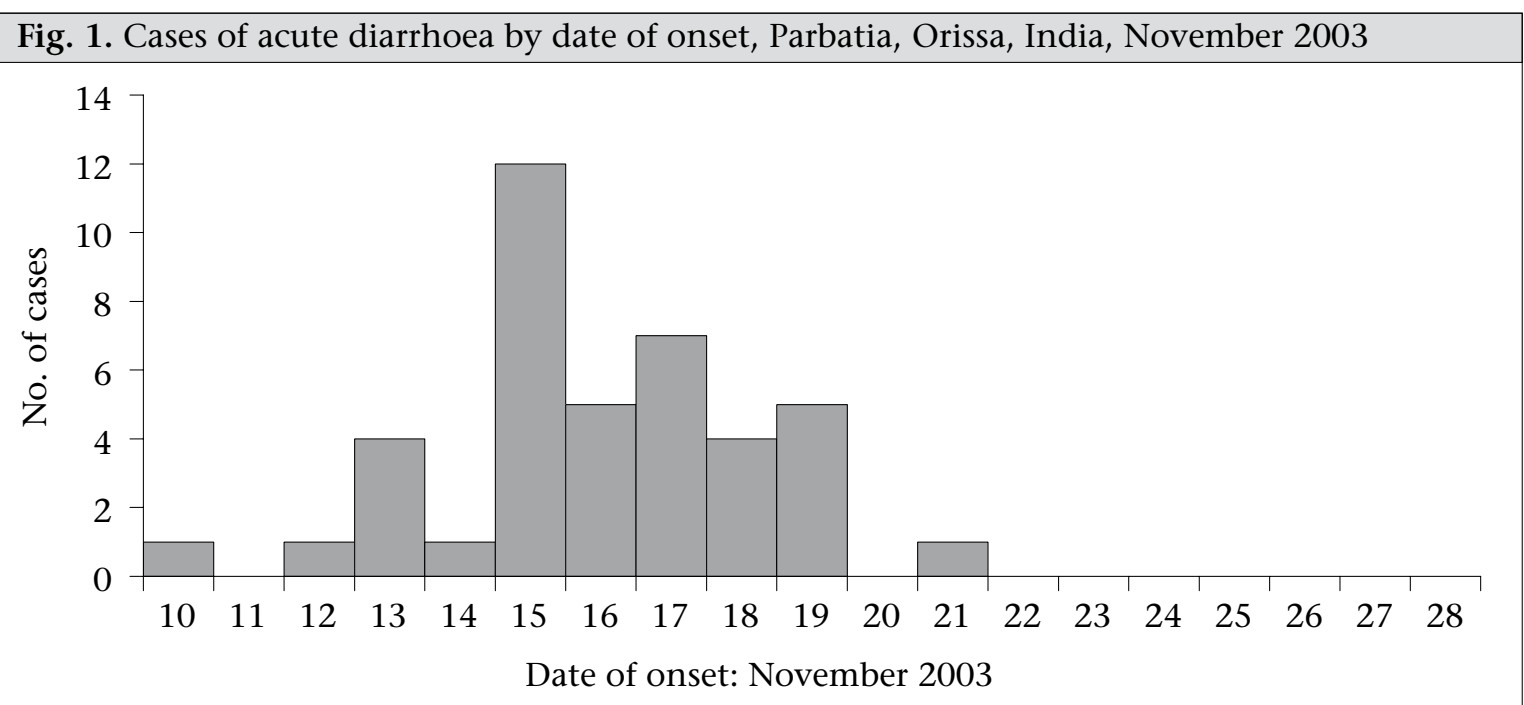




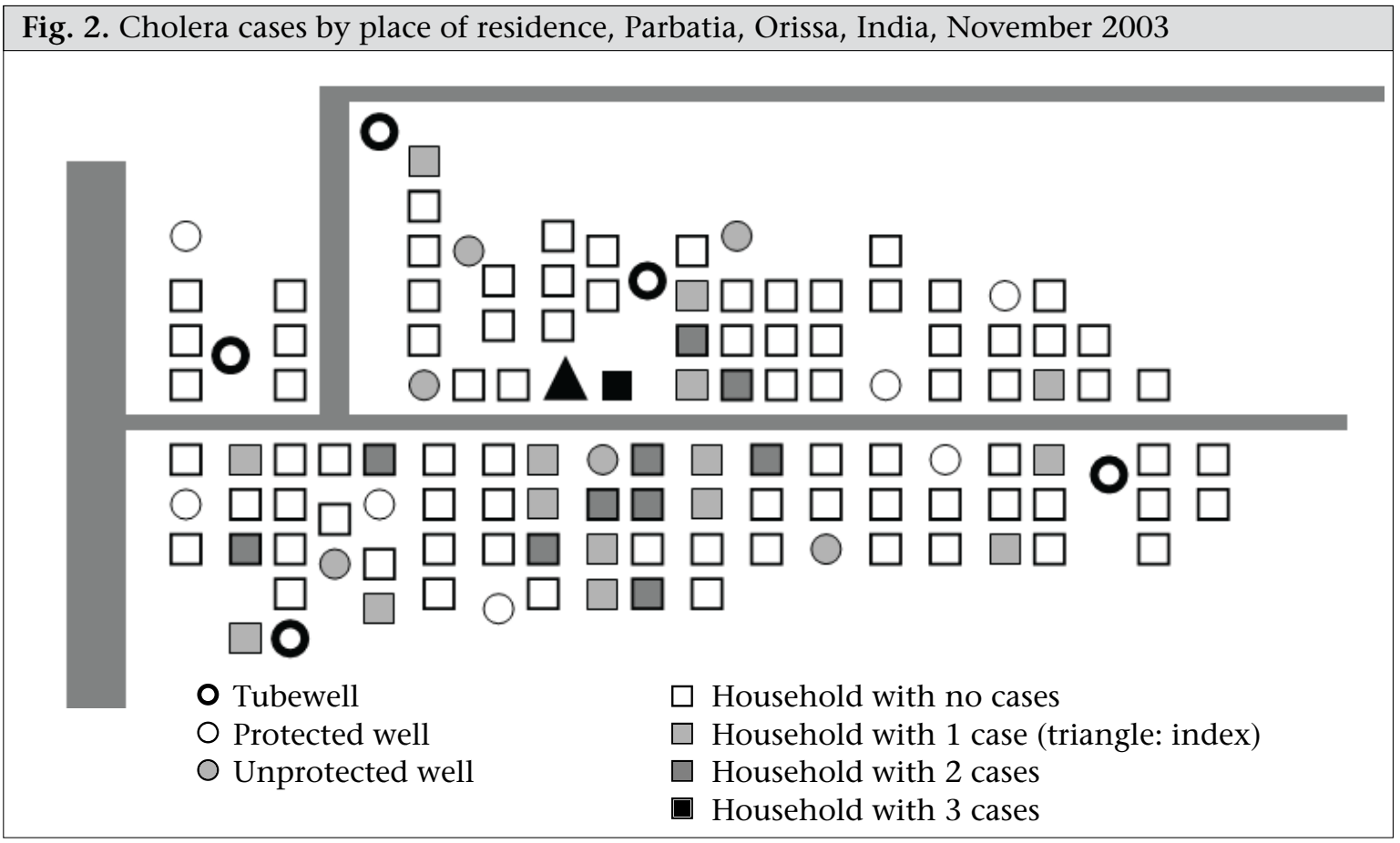

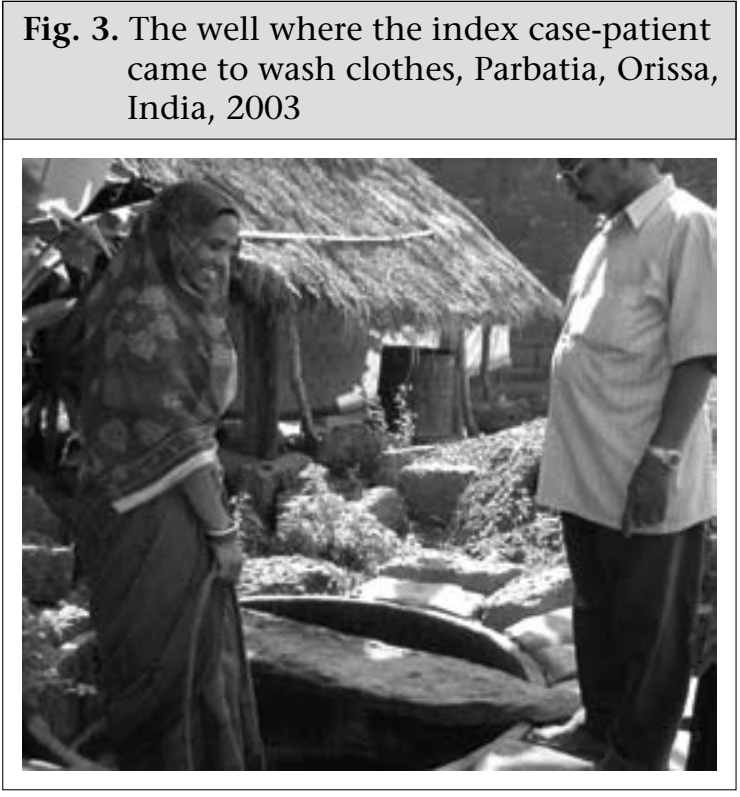

criteria, improved drinking-water sources include household connections, public standpipes, boreholes, protected dugwells, protected springs, and rainwater collection (11). This MDG is challenging for India. In 2001, 64\% of the total population in the state of Orissa and $63 \%$ of the rural population had access to improved water sources. These figures are lower than for the whole of India where 78\% of the total population ( $73 \%$ and $90 \%$ of the rural and urban populations respectively) had access to improved water sources in 2001 (12). Unprotected wells, such as the one that may have caused this outbreak, are not considered improved water sources as per the MDG criteria (11). The occurrence of this outbreak supports the hypothesis that these are dangerous for the population as these can be the source of waterborne epidemic.

The well that we suspected to be the source of infection may have been one of the several sources during this outbreak. The second part of the epidemic curve following the 15 November peak was more stretched than what could be expected from a point source cluster. Furthermore, the attributable fraction suggested that the well only accounted for $64 \%$ of the cases. Thus, during the second part of the outbreak, a number of cases may have been secondary to other modes of transmission, including person-to-person transmission. During outbreaks of cholera, more than one source are commonly involved. Environmental contamination is substantial because of the high concentration of pathogens in stools of patients. However, the well that we identified as a source of infection constituted an initial input that was probably essential to the dynamic of the outbreak, although it might not have accounted for all the cases.

Our investigation had three limitations. First, we only obtained laboratory confirmation for a limited number of case-patients. Thus, we cannot exclude that the outbreak could have been caused by a combination of more than one microorganism. However, our suspicion of cholera was based on the case definition that was compatible with the 
diagnosis of cholera. The diagnosis was confirmed using rectal swab from a public-health perspective. The case-patients with dehydration, irrespective of the laboratory confirmation of the diagnosis, were also treated. Second, we did identify any other specific vehicle in the hypothesis-generation stage and in the case-control analysis. However, the conclusions and recommendations from the investigation would not be different if the agent or vehicle had been different. Third, we did not test the status of subclinical infection among controls. This would have amounted to a non-differential misclassification. This error would only lead to an under-estimation of the strength of the association, which would not affect our conclusion.

Overall, this outbreak allowed the use of applied field epidemiology methods to generate practical information for public-health action. A unique and novel aspect of this outbreak was the use of field epidemiologists in training to recognize the outbreak and to propose and implement measures to control cholera. The field epidemiologist in training was under supervision by NIE-based mentors. Most FETPs globally (including Epidemic Intelligence Service [EIS]), Centers for Disease Control and Prevention, USA) rely on supervised trainees to investigate outbreaks, and investigations of outbreak do not require ethical committee clearance (13).

Our investigations pointed to the danger of unprotected wells and their capacity to spread pathogens, including V. cholerae. Unsafe use of the well to wash clothes contaminated with faeces led to a common source cluster, possibly followed by a certain amount of person-to-person transmission. Based on these conclusions, we formulated a number of recommendations and engaged in a number of interventions. First, we immediately warned the community that the well was dangerous and barred access to it. Second, we chlorinated the well. However, we did not check the impact of that measure. Third, we worked with the village leaders to provide alternative source of water while the use of the well was banned. We also engaged in a number of interventions for long term. First, washing of clothes in the wells was banned. Second, we worked with the rural engineering department to protect the well that had been involved in the outbreak. In June 2004, the safer, redesigned, protected well was available for use again to the population. Third, the population was educated for the safe use of wells so that they would abstain from opportunities of contaminations, such as the one that occurred during this outbreak. Fourth, we worked with the district authorities to make sure that all new wells constructed locally would be protected. Availability of evidence from this outbreak helped us convince them and facilitated the investment of additional resources to build protected wells. This investigation is comparable with that of John Snow's classical studies and the removal of the pump-handle (14). In fact, this FETP work became a case study in the Indian FETP programme and is used by the WHO to teach investigation of outbreaks.

To summarize, more safer, protected wells will be needed in Orissa and in India to control cholera in the longer term. Standardized indicators of the millennium will allow monitoring progress towards these objectives in the future. The broader use of field epidemiology methods should also help us track the various modes of transmission of cholera in the country so that all modes of transmission are addressed with adapted, evidence-based interventions.

\section{REFERENCES}

1. Miller CJ, Feachem RG, Drasar BS. Cholera epidemiology in developed and developing countries: new thoughts on transmission, seasonality, and control. Lancet 1985;1:261-2.

2. Tauxe RV, Mintz ED, Quick RE. Epidemic cholera in the new world: translating field epidemiology into new prevention strategies. Emerging Infect Dis 1995;1:141-6.

3. World Health Organization. Guidelines for cholera control. Geneva: World Health Organization, 1993. $68 \mathrm{p}$.

4. Sur D, Dutta S, Sarkar BL, Manna B, Bhattacharya MK, Datta KK et al. Occurrence, significance \& molecular epidemiology of cholera outbreaks in West Bengal. Indian J Med Res 2007;125:772-6.

5. Ramamurthy T, Garg S, Sharma R, Bhattacharya SK, Nair GB, Shimada T et al. Emergence of novel strain of Vibrio cholerae with epidemic potential in southern and eastern India. Lancet 1993;341:703-4.

6. Singh J, Bora D, Khanna KK, Jain DC, Sachdeva V, Sharma RS et al. Epidemiology and transmission of $V$. cholerae $\mathrm{O} 1$ and V. cholerae O139 infections in Delhi in 1993. J Diarrhoeal Dis Res 1996;14:182-6.

7. Zuckerman JN, Rombo L, Fisch A. The true burden and risk of cholera: implications for prevention and control. Lancet Infect Dis 2007;7:521-30.

8. Rothenberg RB, Hahn RA. Appendix J: Measures of attribution. In: Haddix AC, Teutsch SM, Shaffer PA, Dunet DO, editors. Prevention effectiveness: a guide to decision analysis and economic evaluation. New 
York, NY: Oxford University Press, 1996:193-202.

9. Hamner S, Tripathi A, Mishra RK, Bouskill N, Broadaway SC, Pyle BH et al. The role of water use patterns and sewage pollution in incidence of water-borne/ enteric diseases along the Ganges river in Varanasi, India. Int J Environ Health Res 2006;16:113-32.

10. Sur D, Dutta P, Nair GB, Bhattacharya SK. Severe cholera outbreak following floods in a northern district of West Bengal. Indian J Med Res 2000;112:178-82.

11. WHO/UNICEF Joint Monitoring Programme for Water Supply and Sanitation. Meeting the MDG drinking water and sanitation target: a mid-term assessment of progress. New York, NY: United Nations
Children's Fund, 2004. 33 p.

12. Access to safe drinking-water in house holds in India. (indiabudget.nic.in/es2006-07/chapt2007/tab96.pdf, accessed on 20 April 2008).

13. Thacker SB, Dannenberg AL, Hamilton DH. Epidemic intelligence service of the Centres for Disease Control and Prevention: 50 years of training and service in applied epidemiology. Am J Epidemiol 2001;154:98592.

14. Snow J. On the mode of communication of cholera. In: Snow on cholera. 2nd ed. New York, NY: Hafner Publishing Co., 1965 (reprint). (http://www.ph.ucla. edu/epi/snow.html, accessed on 20 April 2008). 